\title{
Reviewer Summary
}

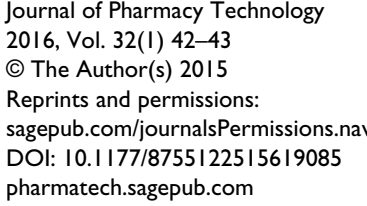

(S)AGE

The individuals listed below reviewed manuscripts for the Journal of Pharmacy Technology between November I, 2014 and November I, 2015. We are glad to take this opportunity to acknowledge our referees and to thank them for their time and effort.

Abeyta, Agnieszka

Aguayo, Doreene R

Aikens, Garrett B

Al-Achi, Antoine

Al-Gain, Roaa A

Alexander, Gail Orum

Ali, Saadia

Allen Jr, Loyd

Amerine, Lindsey

Anderson, Sarah

Anderson, Shawn D

Anselmo, Lisa M

Arnold, Lindsay M

Aruru, Meghana

Aslani, Parisa

Awad, Magdi

Awogbemi, Kayode

Babcock, Charles K

Bae, Yuna

Bailey, Stacy

Bajorek, Beata V

Balano, Kirsten

Bandy, Jason L

Bandy, Veronica T

Barreuther, Alan D

Barros, Michael C

Battaglia, Jessica N

Beck, Shawna

Beigi, Pooya

Berg, Melody L

Berlie, Helen D

Biddle, Jr, Michael A

Bohm, Nicole M

Borchert, Jill S

Bork, Sara

Boyce, Eric G

Brittain, Jeff

Brown, Sherrill J

Brummel, Gretchen L

Brunetti, Luigi

Bucher, Kasey

Bullock, Laura N

Bykov, Katsiaryna
Carr, Glenda

Chen, Tein-Yun

Christensen, Keith J

Christiansen, Blake

Chung, Eun Kyoung

Coppenrath, Valerie

Cottencin, Olivier

Cox, Laura McIntyre

Cummings, Brian M.

Dang, Yalong

Daniels, Heidi S

Darbishire, Patricia L

Daugherty, Kimberly K

DelMonte, Keith

DiMondi, Vincent

Dobson, Erica Lynn

Dong, Yizhou Dong

Dumkow, Lisa

Dunham, Marissa Waldman

Duprey, Matt

Eagerton, David

Edwards, Corey

Edwards, Melinda

Eisenhower, Christine

Enato, Ehijie FO

Faldasz, Jonathan

Fang, Yu

Ferketich, Amy

Fletcher, Lynn

Garcia, Angela

Geddis, Janell

Gibson, Mara

Greenberg, Karin A

Griffiths, Carrie

Gwak, Hye Sun

Hall, Ronald

Hardin, Heather

Hartzler, Melody

Herink, Megan

Herk, Brandon

Hermes-DeSantis, Evelyn R

Hilaire, Michelle L

Hohmeier, Kenneth 
Horton, Evan R

Hughes, Christine A

Huston, Sally A

Ivy, Delaney

John, Barnabas M

Johnson, Jessica L

Johnson, Thomas

Jordan, Melanie

Justis, Leanne

Kelly, Michael S.

Kim, Eun Young

King, Benjamin

Kissling, Kevin

Knezevich, Jon

Kopcza, Kathleen B

Krajewski, Kristin

Kuester, Melanie K

Lacher, Barbara

Lai, Francine Fusee

LaVance, Anne

Law, Anandi V

LeBlanc, Yvonne C

Levien, Terri L

Lloyd, Stanley J.

Lopez, Julio R

Lyles, Adraine Lawrence

Makowski, Charles

Marrero, Abby

Mersfelder, Tracey L

Molloy, Leah

Moore, Troy

Mospan, Cortney

Mospan, Cortney

Murray, Kyle P

Musil, Elizabeth

Nelson, Agatha Christie

Nelson, Donald

Nguyen, Theresa

paparoupa, maria

Pappas, Ashley L

Parker, Lois

Parsons, Kara

Patel, Sruti R

Peeters, Michael J

Pogge, Elizabeth K

Ponder, Richard

Prosser, Theresa
Reeve, Emily

Rhee, Diane H

Rice, Kathryn

Rieder, Michael J.

Rieser, Kristin

Rivard, Kaitlyn

Ruck, Bruce

Scholl, Justin D

Schommer, Jon C

Schulz, Martin

Scolaro, Kelly L

Shea, Peter

Singh-Franco, Devada

Sirois, Caroline

Snyder, Mitchell

Sonawane, Kalyani

Stender, Jennifer

Stultz, Jeremy

Su, Mark

Tajchman, Sharla K

Thomas, Michael JW

Thomason, Angela R

Tsoukleris, Mona

Vogel Anderson,

Katherine

Waghel, Rashi C.

Waki, Bradd

Wellman, Jessica C

Westerlund, LO Tommy

Wheeler, James

William, Jeffrey

Williams, Brett

Wilson, Ashley

Wilson, Dustin

Wilson, James Paul

Wilson, Jennifer

Winders, Timothy

Wingate, LaMarcus

Wood, G Christopher

Wright, Bradley

Yiannakopoulou, Eugenia

Yoon, Hyonok

Zell-Kanter, Michele

Zito, Annapaola

Zomp, Amanda

Zorek, Joseph A

Zukkoor, Sarah M 